\title{
Formulation, Characterization and Stability of Ibuprofen-Loaded Self- Nano Emulsifying Drug Delivery System (SNEDDS)
}

\author{
Yandi Syukri*, Hannie Fitriani, Herianto Pandapotan and Bambang Hernawan Nugroho \\ Department of Pharmacy, Universitas Islam Indonesia Jl. Kaliurang KM 14.5 Yogyakarta, Indonesia 55584
}

\author{
Info Article \\ Submitted: 01-02-2019 \\ Revised: 30-04-2019 \\ Accepted: 22-05-2019 \\ *Corresponding author \\ Yandi Syukri \\ Email: \\ yandisyukri@uii.ac.id
}

\begin{abstract}
Ibuprofen is a poorly water-soluble drug with analgesic, antipyretic and anti-inflammatory effects. Self-Nano Emulsifying Drug Delivery System (SNEDDS) formulation is a solution to improve the solubility and bioavailability of ibuprofen. This research purposed to perform a formulation, characterization, and stability studies of ibuprofen-loaded SelfNano Emulsifying Drug Delivery System (SNEDDS). Screening of ibuprofen SNEDDS was prepared by ternary diagrams for the chosen co-surfactants, surfactants, and oil. The following was characterizations of droplet size, zeta potential, and clarity. The solubility test for the determination of cosurfactant, surfactant, and oil obtained Propylene glycol monocaprylate (Capryol-90), Polysorbate 20 (Tween 20) and PEG 400. The screening of SNEDDS showed nine formulas (compositions) in the range concentration of Propylene glycol monocaprylate (1-3mL), Polysorbate 20 (4-8mL), and PEG 400 (1-3mL). The composition of Propylene glycol monocaprylate (1-2mL), Polysorbate $20(5-8 \mathrm{~mL})$ and PEG $400(1-3 \mathrm{~mL})$ passed the thermodynamic stability test. The test of robustness to dilution and stability study indicated that the formula with Propylene glycol monocaprylate, Polysorbate 20 and PEG 400 with the ratio of 1: 8: 1 and 1: 7: 2 was more stable. In conclusion, the stable ibuprofen SNEDDS could be prepared with Propylene glycol monocaprylate, Polysorbate 20, and PEG 400.
\end{abstract}

Keywords: Ibuprofen, SNEDDS, characterization, stability

\section{INTRODUCTION}

Well-known as an antipyretic, analgesic, and non-steroidal drug for anti-inflammation, ibuprofen is useful for fever, its symptoms, and rheumatoid arthritis and osteoarthritis signs. Due to its low solubility and high permeability, ibuprofen is categorized as BCS class II (Álvarez et al., 2011; Deng et al., 2018). The solubility and bioavailability of ibuprofen should be increased to improve therapy effectiveness by developing the drug delivery system. Among the approaches to enhance dissolution characteristics of waterinsoluble drugs are cyclodextrin complexation, particle-size reduction, crystal modification, and self-emulsification (Syukri et al., 2015). Meanwhile, self-micro emulsifying, self-emulsifying, and liposomes are among the most widely-developed methods to improve solubility and bioavailability of orally-given drugs in lipid-based formulations (Shafiq-un-Nabi et al., 2007).

Some common permeability enhancers include lipid-based excipients, such as fatty acids and glycerides as well as ionic and non-ionic surfactants. Such excipients can increase membrane fluidity or open tight junctions (Kuentz, 2012). The entry of some highly lipophilic drugs and long-chain triglycerides (TG) into the portal circulation, however, is reduced depending on their entry into the lymphatic circulation. Hence, lymphatic- rather than portal-transported drugs can avoid the first-pass metabolism by the liver that in turn enhances the concentration of drug in lymph ducts and nodes where therapeutic drug action exists (Yáñez, et al., 2011).

Self-Nano Emulsifying Drug Delivery System (SNEDDS) becomes one of these simplified approaches to enhance drug solubility and dissolution. In SNEDDS, a thermodynamically stable oil-in-water emulsion is formed spontaneously when an isotropic mixture of drug, oil, surfactant, and co-surfactant enters the aqueous phase under gentle agitation conditions resembling those to be encountered in the GI tract (Dash, et al., 2015a; Sriamornsak, et al., 2015). 
Table I. Composition of oil, surfactant, and co-surfactant for construction of ternary diagrams

\begin{tabular}{cccc}
\hline Composition & 0il (mL) & Surfactant $(\mathbf{m L})$ & Co-surfactant $(\mathbf{m L})$ \\
\hline C1 & 1 & 8 & 1 \\
C2 & 1 & 7 & 2 \\
C3 & 1 & 6 & 3 \\
C4 & 2 & 7 & 1 \\
C5 & 2 & 6 & 2 \\
C6 & 2 & 5 & 3 \\
C7 & 3 & 6 & 1 \\
C8 & 3 & 5 & 2 \\
C9 & 3 & 4 & 3 \\
C10 & 4 & 5 & 1 \\
C11 & 4 & 4 & 2 \\
C12 & 4 & 3 & 3 \\
C13 & 5 & 4 & 1 \\
C14 & 5 & 3 & 2 \\
C15 & 5 & 2 & 3 \\
\hline
\end{tabular}

Studies show that SNEDDS improve the solubility, dissolution, and bioavailability of drugs with poor solubility in water, including lovastatin (Yadava, et al., 2015), glipizide (Agrawal, et al., 2015), nisoldipine (Nekkanti et al., 2016), and anti-cancer genistein (Shehata, et al., 2016). The SNEDDS of nystatin was found to be able to improve dissolution as well as oral antifungal efficacy (Kassem et al., 2016). Another study revealed that, compared to the plain formulation, the andrographolide isolated from Andrographis paniculata plant in the form of SNEDDS formulation provided better bioavailability and dissolution (Syukri et al., 2018).

Ibuprofen is currently marketed commercially in tablet and capsule dosage forms. Both forms demonstrate low, erratic oral bioavailability because of their poor solubility and dissolution (Shahba et al., 2012). Ibuprofen can be formulated into SNEDDS to enhance absorption as well as avoid the first-pass metabolism. This study aimed to prepare, characterize, and examine the stability of ibuprofen in SNEDDS formulation.

\section{MATERIALS AND METHODS Materials}

Ibuprofen was bought from Cross Chem. Polysorbate 80 (Tween 80), Polysorbate 20 (Tween 20), oleic acid, olive oil, polyethylene glycol (PEG) 400, and propylene glycol were obtained from Brataco Indonesia Ltd. Labrasol, Propylene glycol monocaprylate (Capryol 90), and Labrafil
M1944CS were gifted by Gattefose (France), Cremophor RH 40 from BASF (Indonesia), and Myritol 318 from Phapros Indonesia Ltd.

\section{Solubility test to select oil, surfactant, and co-} surfactant

The shake-flask method was used to determine ibuprofen solubility in different oil, surfactant, and co-surfactant concentrations. Extra ibuprofen was added into each vehicle and mixed in the vortex (Heidolph Reax Top, Germany) for $10 \mathrm{~min}$ followed by a 48 -h mixture shaking at $37^{\circ} \mathrm{C}$ in a shaking water bath with thermostaticallycontrolled condition from Memmert WNE 45, Germany. After reaching stability for $24 \mathrm{~h}$, the mixture was put in centrifugation from Hettich Mikro 22R, Germany at $6000 \mathrm{rpm}$ for $10 \mathrm{~min}$. The supernatant was collected, and concentration was calculated employing UV-Vis spectrophotometer (Shimadzu UV 1800, Japan) at a wavelength of 223nm.

\section{Construction of ternary diagrams}

The selected co-surfactants, surfactants, and oils obtained from the solubility study were plotted in a ternary diagram without incorporation of the drug; one side of the triangle represented each selection for identification of the region of selfnano emulsifying (Kassem et al., 2016). A set of oil, surfactant, and co-surfactant mixtures was provided with composition of 1-5; 2-8; and 1-3mL, respectively. The composition of oil, surfactant, and co-surfactant for ternary phase diagrams (Table I). 
The assessment was conducted for the nanoemulsion formed after each formulation (compositions) was given 100-fold dilution in aquabidest. The clarity, zeta potential, and droplet size of resulted dispersions were then measured using particle size analyzer from Horiba SZ 100; Japan and UV-Vis spectrophotometer from Shimadzu UV 1800; Japan at a wavelength of $650 \mathrm{~nm}$. Dispersions remained up to standard as long as the droplet size was not more than $200 \mathrm{~nm}$. Then, the ternary phase diagrams of co-surfactant, surfactant, and oil were plotted.

\section{Preparation of ibuprofen-loaded SNEDDS}

As much as $5 \mathrm{~mL}$ mixture of oil, surfactant, and co-surfactant was used to dissolve Ibuprofen (200mg) for preparation of SNEDDS formulation. Dissolution was conducted in ultrasonic homogenizer (model $300 \mathrm{~V} / \mathrm{T}$, USA) for solubilization to obtain clear solution. The mixture was then stored at ambient temperature for future use.

\section{Ibuprofen-loaded SNEDDS characterization Percentage of clarity (transmittance) studies \\ Purified water was used to dilute the} ibuprofen-loaded SNEDDS formulations 100 times. The clarity (\%) was determined through UV-Vis spectrophotometer from Shimadzu UV 1800, Japan at a wavelength of $650 \mathrm{~nm}$ (Nagi et al., 2017), and the blank was purified water.

\section{Zeta potential and droplet size}

The zeta potential and droplet size of SNEDDS were measured by particle size analyzer from Horiba SZ 100; Japan followed by dilution of SNEDDS samples in purified water for 100 times.

\section{Thermodynamic stability studies Centrifugation study}

Centrifugation of the formulations was conducted at $5000 \mathrm{rpm}$ for $30 \mathrm{~min}$ followed by visual observation to confirm instability, including cracking, phase separation, drug precipitation, or creaming. The formulations without any signs of weakness were selected for the heating-cooling test.

\section{Heating and cooling cycle}

The heating and cooling cycle was done in three repetitions at a temperature range of $4^{\circ} \mathrm{C}-45^{\circ} \mathrm{C}$ and a minimum of 48 -hour storage. The period was conducted in one repetition for $8 \mathrm{~h}$ at a temperature of $4^{\circ} \mathrm{C}$ and $8 \mathrm{~h}$ at a temperature of $45^{\circ} \mathrm{C}$. The formulations that survived such condition without signs of creaming and phase separation were selected for the freezing and thawing cycle.

\section{Freezing and thawing cycle}

The freeze and thaw test was conducted 3 times between $-20^{\circ} \mathrm{C}$ and $+25^{\circ} \mathrm{C}$ temperature with a minimum of 48 hours storage duration. The period was held in one repetition for $8 \mathrm{~h}$ at a temperature of $4^{\circ} \mathrm{C}$ and $8 \mathrm{~h}$ at a temperature of $45^{\circ} \mathrm{C}$. Further analysis was performed for the formulations surviving such thermodynamic stress test (Agrawal et al., 2015; Kassem et al., 2016).

\section{Robustness to dilution}

Robustness to dilution significantly affects the phase separation of a spontaneously emulsifying system. A system with spontaneous emulsification attempts to emulate in vivo conditions where formulations dilute g radually. Dilution of ibuprofen-loaded SNEDDS in purified water was conducted for 25, 50, 100, and 250 times. The droplet size was then evaluated to select the compositions with consistent droplet size after dilution in different dispersion media to proceed with in-vitro assay (Balakumar, et al., 2013; Elnaggar et al., 2009). Milky-like composition was let rest while the transparent one was observed to determine the droplet size.

\section{Stability study}

To assess the accelerated stability, the optimized ibuprofen-loaded SNEDDS was stored in sealed amber glass vials for four weeks at a high temperature $\left(40^{\circ} \pm 2^{\circ} \mathrm{C}\right)$ and relative humidity $(75 \pm 5 \%)$. The changes over time in the physical characteristics, such as drug droplet size and clarity, were monitored to evaluate the physical stability of the optimized composition. The oneway ANOVA was used for comparing droplet size changes every week.

\section{RESULTS AND DISCUSSION}

Solubility test to select oil, surfactant, and cosurfactant

Drug solubility in vehicles is essential to determine formulation stability because it is commonly found that many formulations precipitate before experiencing in situ solubilization. A combination of oil, surfactant, and co-surfactant produce a self-emulsifying mixture. Such mixture should provide a transparent and 
Table II. Solubility test of ibuprofen in varied oils, surfactants, and co-surfactants (n=3)

\begin{tabular}{ccc}
\hline Vehicle & Material & Solubility $\mathbf{( m g} / \mathbf{m L})$ \\
\hline \multirow{3}{*}{ Oil } & Propylene glycol monocaprylate & $64.53 \pm 0.19$ \\
& Myriol 318 & $13.33 \pm 0.01$ \\
& Oleic acid & $60.67 \pm 0.09$ \\
& Olive oil & $9.53 \pm 0.01$ \\
Surfactant & Labrasol & $83.18 \pm 0.74$ \\
& Labrafil M1944CS & $26.18 \pm 0.02$ \\
& Polysorbate 20 & $97.71 \pm 0.16$ \\
Co-surfactant & Polysorbate 80 & $73.10 \pm 0.16$ \\
& Cremophor RH 40 & $36.55 \pm 0.14$ \\
& PEG 400 & $35.02 \pm 0.08$ \\
& Propylene glycol & $32.34 \pm 0.09$ \\
\hline
\end{tabular}

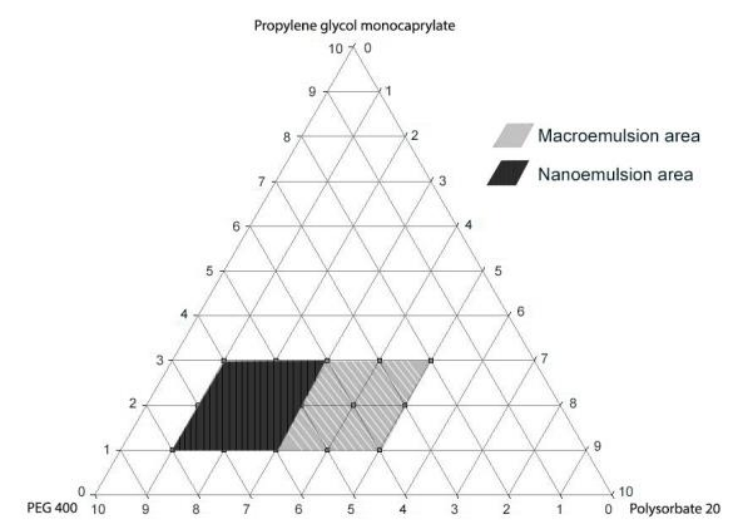

Figure 1. Ternary diagram of ibuprofen showing the region of o/w nanoemulsion in Propylene glycol monocaprylate, Polysorbate 20 and PEG 400 as the oil, surfactant and co-surfactant

isotropic liquid at a room temperature with fine solubility to integrate a dose of drug in a minimum volume of mixture (Kassem et al., 2016)

Oils, surfactants, and co-surfactants for SNEDDS formulations are selected according to the highest solubility of ibuprofen in each of them. Besides, the selection is also based on the ability of the mixtures to form nanoemulsion when diluted 100 times with aquabidest. The results of ibuprofen solubility test in different sets of oil, surfactant, and co-surfactant (Table II).

Table II indicates that the oil phase, Propylene glycol monocaprylate, had the best solubilizing capacity $(64.53 \pm 0.19)$, and for the same reason Polysorbate $20(97.71 \pm 0.16)$ and PEG $400 \quad(35.02 \pm 0.08)$ were chosen as the best surfactant and co-surfactant.

\section{Construction of ternary diagrams}

The self-nano emulsifying area is determined using a ternary phase diagram, which is also utilized to assess oil, surfactant, and co- surfactant concentrations for stable SNEDDS. Figure 1 illustrated the ternary phase diagram of formulations with a carrier consisting of Propylene glycol monocaprylate, Polysorbate 20, and PEG 400.

The shaded/dark area marks the regions of nanoemulsion and macroemulsion. The formulation with $1-3 \mathrm{~mL}$ of Propylene glycol monocaprylate (oil), 4-8mL of Polysorbate 20 (surfactant), and 1-3mL of PEG 400 (co-surfactant) has produced transparent nanoemulsion without sedimentation. In such region, spontaneous self-emulsification results in dispersion that is thermodynamically stable. Meanwhile, the composition having $4-5 \mathrm{~mL}$ Propylene glycol monocaprylate produces milkylike or crude macroemulsion (Kassem et al., 2016; Parmar et al., 2011) in which no self-emulsification occurs.

\section{Preparation of ibuprofen-loaded SNEDDS}

The inexistence of precipitation and phase separation for $48 \mathrm{~h}$ indicates that ibuprofen 
Table III. Droplet size, zeta potential, clarity, and description of ibuprofen-loaded SNEDDS as an effect of various oil, surfactant and co-surfactant concentrations ( $\mathrm{n}=3$ )

\begin{tabular}{ccccc}
\hline Composi-tion & Droplet size (nm) & Zeta potential (mV) & Clarity (\%) & Description \\
\hline C1 & $12.63 \pm 1.68$ & $-24.37 \pm 0.29$ & $99.98 \pm 0.01$ & Transparent \\
C2 & $12.00 \pm 2.60$ & $-37.37 \pm 0.64$ & $99.83 \pm 0.01$ & Transparent \\
C3 & $15.20 \pm 1.70$ & $-24.55 \pm 0.29$ & $99.48 \pm 0.04$ & Transparent \\
C4 & $15.00 \pm 0.10$ & $-31.00 \pm 0.52$ & $99.98 \pm 0.01$ & Transparent \\
C5 & $20.97 \pm 1.72$ & $-41.53 \pm 0.58$ & $99.86 \pm 0.01$ & Transparent \\
C6 & $15.76 \pm 1.58$ & $-34.83 \pm 0.68$ & $100.00 \pm 0.00$ & Transparent \\
C7 & $70.53 \pm 3.43$ & $-22.03 \pm 0.55$ & $100.00 \pm 0.00$ & Transparent \\
C8 & $41.33 \pm 3.23$ & $-18.73 \pm 0.51$ & $99.71 \pm 0.02$ & Transparent \\
C9 & $47.60 \pm 1.73$ & $-37.90 \pm 0.61$ & $99.41 \pm 0.01$ & Transparent \\
C10 & $321.67 \pm 12.00$ & $-17.40 \pm 0.36$ & $33.16 \pm 0.02$ & Cloudy \\
C11 & NA & $-30.40 \pm 0.17$ & $33.67 \pm 0.01$ & Cloudy \\
C12 & NA & $-36.10 \pm 0.00$ & $26.77 \pm 0.02$ & Cloudy \\
C13 & $498.70 \pm 19.41$ & $-46.97 \pm 0.29$ & $13.12 \pm 0.00$ & Cloudy \\
C14 & NA & $-63.33 \pm 0.40$ & $20.29 \pm 0.01$ & Cloudy \\
C15 & NA & $-27.20 \pm 0.35$ & $16.77 \pm 0.11$ & Cloudy \\
\hline
\end{tabular}

NA: Not Available (Droplet size was undetected

dissolves in SNEDDS. In this study, ibuprofen was stable in the SNEDDS formulation with $200 \mathrm{mg} / 5 \mathrm{~mL}$ drug loading. The results of the characterization by calculating the droplet size, clarity, and zeta potential (Table III).

\section{Droplet size}

Range of droplet size of C1 to C9 is between $12.00 \pm 2.60 \mathrm{~nm}$ and $70.53 \pm 3.43 \mathrm{~nm}$. C1 to C9 are defined as nanoemulsion because they form a clear dispersion, and since $\mathrm{C} 10$ to $\mathrm{C} 15$ have a milky/cloudy appearance, they are categorized as macroemulsion (Table III). Macroemulsion (C10 to C15) with the larger droplet size was not detected the droplet size using particle size analyzer (Horiba SZ 100) because the equipment only covers the droplet size less than $1000 \mathrm{~nm}$. Nanoemulsion size has a droplet size of less than 200nm (Balakumar, et al., 2013). Oil phase in high concentration will produce a large droplet size.

Meanwhile, a large number of cases indicate that increasing surfactant concentration will decrease droplet size because surfactant molecule localization on the interface of oil-water will stabilize oil droplets. High surfactant concentration will improve the penetration of water into oil droplets that lead to the breakdown of oil droplets and formation of larger droplets (Balakumar, et al., 2013). Small co-surfactant molecules will enhance surfactant function in emulsification as they penetrate the film of surfactant on the interface of oil-water, and small droplet size will reduce interfacial tension and improve the spontaneous process of emulsification. Since the free energy required to produce tiny droplet size in a system with self-emulsification is lacking, oil nanoemulsion will be spontaneously formed in water (Heshmati, et al., 2018).

\section{Clarity}

Nanoemulsion is a water-oil-surfactant system, which forms a complete mixture that is optically isotropic and a solution that is thermodynamically stable. Clarity is measured in a clarity test to maintain the isotropic range. In such an analysis, the dispersion-system transparent level will be obtained from water-solution dispersion absorbance at $650 \mathrm{~nm}$ wavelength. Table 3 showed Composition 1 (C1) to 9 (C9) have clear dispersion with almost $100 \%$ clarity, indicating fulfilment of the nanoemulsion requirement.

On the other hand, C10 to C15 produced a milky-like solution with a clarity value of less than $34 \%$. Compositions with less clarity have a more significant droplet size and result in milky-like macroemulsion. The data showed that aqueous dispersion having low transparency is usually cloudy, and globules of oil have larger dispersion. Nanoemulsion clarity indicates self-emulsification efficacy by identifying dispersion stability in a reproducible or short period. 
Table IV. Thermodynamic stability studies of ibuprofen-loaded SNEDDS as an effect of various oil, surfactant, and co-surfactant concentrations $(n=3)$

\begin{tabular}{cccc}
\hline Composition & Centrifugation & Heating and cooling cycle & Freezing and thawing cycle \\
\hline C1 & Allowed & Allowed & Allowed \\
C2 & Allowed & Allowed & Allowed \\
C3 & Allowed & Allowed & Allowed \\
C4 & Allowed & Allowed & Allowed \\
C5 & Allowed & Allowed & Allowed \\
C6 & Allowed & Allowed & Allowed \\
C7 & Not Allowed & Not Allowed & Not Allowed \\
C8 & Not Allowed & Not Allowed & Not Allowed \\
C9 & Not Allowed & Not Allowed & Not Allowed \\
\hline
\end{tabular}

Table V. Robustness to dilution studies of ibuprofen-loaded SNEDDS ( $\mathrm{n}=3$ )

\begin{tabular}{ccccc}
\hline \multirow{2}{*}{ Composition } & \multicolumn{4}{c}{ Droplet size (nm) } \\
\cline { 2 - 5 } & $\mathbf{1 : 2 5}$ & $\mathbf{1 : 5 0}$ & $\mathbf{1 : 1 0 0}$ & $\mathbf{1 : 2 5 0}$ \\
\hline C1 & $11.30 \pm 0.17$ & $11.00 \pm 0.62$ & $12.63 \pm 1.68$ & $10,50 \pm 0.40$ \\
C2 & $11.43 \pm 0.21$ & $13.43 \pm 2.06$ & $12.00 \pm 2.60$ & $10.47 \pm 1.16$ \\
C3 & $11.60 \pm 0.27$ & $12.13 \pm 0.84$ & $15.20 \pm 1.70$ & $23.93 \pm 1.72$ \\
C4 & $251.93 \pm 5.85$ & $310.80 \pm 8.25$ & $15.00 \pm 0.10$ & $65.17 \pm 2.21$ \\
C5 & $332.10 \pm 6.07$ & $268.13 \pm 5.79$ & $20.97 \pm 1.72$ & $53.43 \pm 1.10$ \\
C6 & NA & NA & $15.76 \pm 1.58$ & $46.50 \pm 5.60$ \\
\hline
\end{tabular}

NA: Not Available (Droplet size was undetected)

\section{Zeta potential}

A high value of zeta potential has better stability since there is resistance to aggregation of the formulation. Zeta potential of less than $-30 \mathrm{mV}$ and more than $+30 \mathrm{mV}$ is generally appropriate for the stability of a system (Dash, et al., 2015b). The nanoemulsion area of C1, C3, C7 and C8 produces a zeta potential of more than $-30 \mathrm{mV}$ $(-24.37 \pm 0.2,-24.55 \pm 0.29,-22.03 \pm 0.55$, and $18.73 \pm 0.51 \mathrm{mV}$, correspondingly), whereas the others result in lower than $-30 \mathrm{mV}$ zeta potential (Table III). It means C1, C3, C7 and C8 fail to meet the criteria of zeta potential value for a system stability. A negative value of zeta potential indicates the existence of free fatty acid, surfactant, and co-surfactant in the formulation. It also shows theconsiderableforceofrepulsion between droplets to prevent aggregation (Syukri et al., 2018).

\section{Thermodynamic stability test}

Kinetic stability is crucial for differentiating nanoemulsion from the emulsion, showing the systems' thermodynamic stability. Nanoemulsion system of SNEDDS is formed following in situ solubilization, and creaming, cracking, or precipitation can be avoided when stability is achieved (Senapati, et al., 2016). For formulations C1 to C9, the study of thermodynamic stability performed include heating and cooling cycle; centrifugation; and freezing and thawing cycle, and then the results (Table IV).

C1 to C6 have no signs of phase separation, turbidity, or drug precipitation (Table IV). Therefore, they are eligible for robustness to dilution test. C7, C8 and C9 did not show a good thermodynamically stability test because these formulae contain a larger of oil compositions. Usually, a larger of the oil composition the droplet size the larger. These conditions tend to cause to the instability system.

\section{Robustness to dilution}

To emulate an in vivo state, SNEDDS compositions are diluted for 25, 50, 100, and 250 times in media. After the dilution process, emulsion uniformity in SNEDDS compositions should remain (Elnaggar et al., 2009). Robustness to dilution test guarantees SNEDDS emulsion uniformity in various frequencies of dilution (Table $\mathrm{V}$ ). Ibuprofen-loaded SNEDDS dilution is conducted 25-, 50-, 100-, and 250-fold using aquabidest, and droplet size is evaluated. 
Table VI. The stability studies optimized ibuprofen-loading SNEDDS formulation ( $\mathrm{n}=3$ )

\begin{tabular}{ccccc}
\hline \multirow{2}{*}{ Week to } & \multicolumn{2}{c}{ C1 } & \multicolumn{2}{c}{ C2 } \\
\cline { 2 - 5 } & Droplet size & Clarity & Droplet size & Clarity \\
\hline 0 & $12.63 \pm 1.68$ & $99.98 \pm 0.01$ & $12.00 \pm 2.60$ & $99.83 \pm 0.01$ \\
1 & $12.70 \pm 1.45$ & $97.87 \pm 0.00$ & $12.67 \pm 1.33$ & $99.04 \pm 0.01$ \\
2 & $14.63 \pm 0.31$ & $97.84 \pm 0.00$ & $14.67 \pm 1.53$ & $99.01 \pm 0.02$ \\
3 & $14.47 \pm 1.89$ & $99.93 \pm 0.00$ & $13.57 \pm 2.02$ & $99.47 \pm 0.02$ \\
4 & $13.63 \pm 1.41$ & $99.89 \pm 0.01$ & $13.23 \pm 0.40$ & $99.73 \pm 0.00$ \\
\hline
\end{tabular}

C1 and C2 with Propylene glycol monocaprylate, Polysorbate 20, and PEG 400 at a ratio of 1: 8: 1 and 1: 7: 2, correspondingly, become the most stable compositions since, after dilution, they have a steady droplet size and are then eligible for accelerated stability test (Table V). A regular droplet size after dilutions shows the possible uniformity of in vivo drug release. Thus, the preparations might have the capability of anticipating gradual dilution in the GI tract (Syukri et al., 2018).

\section{Stability study}

The results of stability studies to optimize ibuprofen-loaded SNEDDS formulations C1 and C2 (Table VI). One-way ANOVA showed that no significant difference $(\mathrm{p}<0.05)$ the droplet size during storage for 4-week. Both preparations are physically stable, and there are no apparent changes in physical appearance, including the droplet size and clarity, during the 4-week storage at a higher temperature $\left(40^{\circ} \mathrm{C} \pm 2^{\circ} \mathrm{C}\right)$ and relative humidity $(75 \pm 5 \%)$. It is then clear that stable preparations are found in the ibuprofen-loaded SNEDDS formulations with Propylene glycol monocaprylate, Polysorbate 20 and PEG 400 as the oil, surfactant and co-surfactant at a ratio of 1: 8: 1 (C1) and 1: 7: 2 (C2).

\section{CONCLUSION}

Ibuprofen was prepared in SNEDDS formulation with Propylene glycol monocaprylate, Polysorbate 20, and PEG 400 as the oil, surfactant, and co-surfactant, respectively. Nine compositions with the concentration of Propylene glycol monocaprylate (1-3mL), Polysorbate 20 (4-8mL), and PEG 400 (1-3mL) were the optimal formulation to form a nanoemulsion. The formulation with the ratio of 1: 8: 1 and 1: 7: 2 of Propylene glycol monocaprylate, Polysorbate 20 and PEG 400 were selected compositions after the study of thermodynamic stability, robustness to dilution, and accelerated stability. These evaluations showed the droplet size between $10.50 \pm 0.40$ $13.43 \pm 2.06 \mathrm{~nm}$ after dilution and $12.00 \pm 2.60$ $19.67 \pm 1.53 \mathrm{~nm}$ after accelerated stability studies.

\section{ACKNOWLEDGEMENTS}

The authors are grateful to the Directorate of Research and Community Services and the Pharmaceutical Laboratory of the Department of Pharmacy of Universitas Islam Indonesia for facilitating the research.

\section{REFERENCES}

Agrawal AG., Kumar A. and Gide PS. 2015. Selfemulsifying drug delivery system for enhanced solubility and dissolution of glipizide. Colloids and Surfaces $B$ : Biointerfaces, 126, 553-560. doi.org/10.1016/j.colsurfb.2014.11.022

Álvarez C., Núñez I., Torrado JJ., Gordon J., Potthast H. and García-Arieta A. 2011. Investigation on the Possibility of Biowaivers for Ibuprofen. J Pharm Scie, 100(6), 2343-2349. doi.org/10.1002/jps.22472

Balakumar K., Raghavan CV., selvan NT., Prasad RH. and Abdu S. 2013. Self-nanoemulsifying drug delivery system (SNEDDS) of Rosuvastatin calcium: Design, formulation, bioavailability and pharmacokinetic evaluation. Colloids and Surfaces B: Biointerfaces, 112, 337-343. doi.org/10.1016/j.colsurfb.2013.08.025

Balakumar K., Vijaya Raghavan C., Tamil Selvan N., and Rahman HSM. 2013. Self-emulsifying drug delivery system: Optimization and its prototype for various compositions of oils, surfactants and co-surfactants. J Pharm Res, $6(5)$,

510-514. doi.org/10.1016/j.jopr.2013.04.031

Dash RN., Mohammed H., Humaira T. and Ramesh D. 2015a. Design, optimization and evaluation of glipizide solid self- 
nanoemulsifying drug delivery for enhanced solubility and dissolution. Saudi Pharm J, 23(5),

528-540. https://doi.org/10.1016/j.jsps.2015.01.024

Dash RN., Mohammed H., Humaira T. and Ramesh D.2015b.Design, optimizationand evaluation of glipizide solid self-nanoemulsifying drug delivery for enhanced solubility and dissolution. Saudi Pharm J, 23(5), 528-540. https://doi.org/10.1016/j.jsps.2015.01.024

Deng Y., Yang F., Zhao X., Wang L., Wu W., Zu C. and Wu M. 2018. Improving the skin penetration and antifebrile activity of ibuprofen by preparing nanoparticles using emulsion solvent evaporation method. European J. Pharm. Scie., 114, 293-302. https://doi.org/10.1016/j.ejps.2017.12.024 Elnaggar YSR., El-Massik MA. and Abdallah OY. 2009. Self-nanoemulsifying drug delivery systems of tamoxifen citrate: Design and optimization. International Journal of Pharmaceutics, 380(1-2), 133-141. doi.org/10.1016/j.ijpharm.2009.07.015

Heshmati N., Cheng X., Eisenbrand G. and Fricker G. 2013. Enhancement of Oral Bioavailability of E804 by Self-Nanoemulsifying Drug Delivery System (SNEDDS) in Rats. Journal of Pharmaceutical Sciences, 102(10), 37923799. https://doi.org/10.1002/jps.23696

Kassem AA., Mohsen AM., Ahmed RS. and Essam TM. 2016. Self-nanoemulsifying drug delivery system (SNEDDS) with enhanced solubilization of nystatin for treatment of oral candidiasis: Design, optimization, in vitro and in vivo evaluation. Journal of Molecular Liquids, 218, 219-232. doi.org/10.1016/j.molliq.2016.02.081

Kuentz M. 2012. Lipid-based formulations for oral delivery of lipophilic drugs. Drug Discovery Today: Technologies, 9(2), e97-e104. doi.org/10.1016/j.ddtec.2012.03.002

Nagi A., Iqbal B., Kumar S., Sharma S., Ali J. and Baboota S. 2017. Quality by design based silymarin nanoemulsion for enhancement of oral bioavailability. Journal of Drug Delivery Science and Technology, 40, 35-44. doi.org/10.1016/j.jddst.2017.05.019

Nekkanti V., Rueda J., Wang Z. and Betageri GV. 2016. Comparative evaluation of proliposomes and self-micro emulsifying drug delivery system for improved oral bioavailability of nisoldipine. Intl J Pharm, 505(1-2), doi.org/10.1016/j.ijpharm.2016.03.065
Parmar N., Singla N., Amin S. and Kohli K. 2011. Study of cosurfactant effect on nanoemulsifying area and development of lercanidipine loaded (SNEDDS) selfnanoemulsifying drug delivery system. Colloids and Surfaces B: Biointerfaces, 86(2), 327-338.

doi.org/10.1016/j.colsurfb.2011.04.016

Senapati PC., Sahoo SK. and Sahu AN. 2016. Mixed surfactant based (SNEDDS) selfnanoemulsifying drug delivery system presenting efavirenz for enhancement of oral bioavailability. Biomedicine \& Pharmacotherapy, $\quad 80, \quad 42-51$. doi.org/10.1016/j.biopha.2016.02.039

Shafiq-un-Nabi S., Shakeel F., Talegaonkar S., Ali J., Baboota S., Ahuja A., and Ali M. 2007. Formulation development and optimization using nanoemulsion technique: A technical note. AAPS PharmSciTech, 8(2), E12-E17. https://doi.org/10.1208/pt0802028

Shahba AA.-W., Mohsin K. and Alanazi FK. 2012. Novel Self-Nanoemulsifying Drug Delivery Systems (SNEDDS) for Oral Delivery of Cinnarizine: Design, Optimization, and InVitro Assessment. AAPS PharmSciTech, 13(3), 967-977. doi.org/10.1208/s12249012-9821-4

Shehata EMM., Elnaggar YSR., Galal S. and Abdallah O. Y. 2016. Self-emulsifying phospholipid pre-concentrates (SEPPs) for improved oral delivery of the anti-cancer genistein: Development, appraisal and ex-vivo intestinal permeation. International Journal of Pharmaceutics, 511(2), 745-756. doi.org/10.1016/j.ijpharm.2016.07.078

Sriamornsak P., Limmatvapirat S., Piriyaprasarth, S., Mansukmanee P. and Huang Z. 2015. A new self-emulsifying formulation of mefenamic acid with enhanced drug dissolution. Asian J Pharm.l Scie., 10(2), 121127. doi.org/10.1016/j.ajps.2014.10.003

Syukri Y., Fernenda L., Utami FR., Qiftayati I., Kusuma AP., and Istikaharah R. 2015. Preparation and Characterization of $\mathrm{B}$ Cyclodextrin Inclusion Complexes Oral Tablets Containing Poorly Water Soluble Glimipiride Using Freeze Drying Method. Indonesian Journal of Pharmacy, 26(2), 71. https://doi.org/10.14499/indonesianjphar m26iss2pp71

Syukri Y., Martien R., Lukitaningsih E. and Nugroho A. E. 2018. Novel Self-Nano Emulsifying Drug Delivery System (SNEDDS) of 
andrographolide isolated from Andrographis paniculata Nees: Characterization, in-vitro and in-vivo assessment. Journal of Drug Delivery Science and Technology, 47, 514-520. doi.org/10.1016/j.jddst.2018.06.014

Yadava SK., Naik JB., Patil JS., Mokale VJ. and Singh R. 2015. Enhanced solubility and bioavailability of lovastatin using stabilized form of self-emulsifying drug delivery system. Colloids and Surfaces A: Physicochem. and Engineering Aspects, 481, 63-71. doi.org/10.1016/j.colsurfa.2015.04.026

Yáñez JA., Wang SW. J., Knemeyer IW., Wirth MA. and Alton KB. 2011. Intestinal lymphatic transport for drug delivery. Advanced Drug Delivery Reviews, 63(10-11), 923-942. doi.org/10.1016/j.addr.2011.05.019 